\title{
Interaction matrix element fluctuations in ballistic quantum dots: random wave model
}

\author{
L. Kaplan ${ }^{1}$ and Y. Alhassid ${ }^{2}$ \\ ${ }^{1}$ Department of Physics, Tulane University, New Orleans, Louisiana 70118, USA \\ ${ }^{2}$ Center for Theoretical Physics, Sloane Physics Laboratory, Yale University, New Haven, CT 06520, USA
}

\begin{abstract}
We study matrix element fluctuations of the two-body screened Coulomb interaction and of the one-body surface charge potential in ballistic quantum dots. For chaotic dots, we use a normalized random wave model to obtain analytic expansions for matrix element variances and covariances in the limit of large $k L$ (where $k$ is the Fermi wave number and $L$ the linear size of the dot). These leading-order analytical results are compared with exact numerical results. Both two-body and onebody matrix elements are shown to follow strongly non-Gaussian distributions, despite the Gaussian random nature of the single-electron wave functions.
\end{abstract}

PACS numbers: 73.23.Hk, 05.45.Mt, 73.63.Kv, 73.23.-b

\section{INTRODUCTION}

There has been much interest in the properties of quantum dots whose single-particle dynamics are chaotic [1]. The generic fluctuation properties of the single-particle spectrum and wave functions in such dots are usually described by random matrix theory (RMT) [2]. In open dots that are strongly coupled to leads, electrons can often be treated as non-interacting quasi-particles, and the mesoscopic fluctuations of the conductance have been explained using RMT.

However, in almost-isolated dots, electron-electron interactions are important and must be taken into account. The simplest model of such dots is the constant interaction (CI) model, in which the interaction is taken to be the classical charging energy. Charging energy leads to Coulomb blockade peaks in the conductance versus gate voltage. Each peak occurs as the gate voltage is tuned to compensate for the Coulomb repulsion and an additional electron tunnels into the dot. For a fixed number of electrons, the CI model is essentially a single-particle model, and RMT can be used to derive the statistical properties of the conductance peak heights [3]. While the CI plus RMT model has explained (at least qualitatively) [3, 4, 5] several observed features of the peak height fluctuations [6, 7, 8], there have been significant discrepancies with experimental data, in particular regarding the peak spacing statistics $[9,10,11,12]$. Such discrepancies indicate the importance of interactions beyond charging energy.

A more systematic way of treating electron-electron interactions in chaotic ballistic dots is to expand the interaction in a small parameter, the inverse of the Thouless conductance $g_{T} \sim k L$, where $k$ is the Fermi wave number and $L$ is the linear size of the dot. The Thouless conductance measures the number of single-particle levels within an energy window determined by the time it takes the electron to cross the dot, and $g_{T}$ increases as the square root of the number of electrons. It can be shown that, in the limit of large Thouless conductance, only a few interaction terms survive, constituting the interacting part of the universal Hamiltonian [13, 14]. These uni- versal interaction terms include, in addition to charging energy, a constant exchange interaction. The inclusion of an exchange interaction has explained the statistics of peak heights at low and moderate temperatures as well as the suppression of the peak spacing fluctuations [15, 16]. However, at low temperatures, the peak spacing distribution remains bimodal even when the exchange interaction is included, while none of the experimental distributions are bimodal [9, 10, 11, 12].

For finite Thouless conductance, residual interactions beyond the universal Hamiltonian must be taken into account. The randomness of the single-particle wave functions induces randomness in the two-body screened Coulomb interaction matrix elements [17]. The possible induced two-body ensembles have been classified according to their underlying space-time symmetries and features of the two-body interaction [18]. In a HartreeFock-Koopmans [19] approach (assuming the HartreeFock single-particle wave functions do not change as electrons are added to the dot), the peak spacing can be expressed directly in terms of certain diagonal interaction matrix elements [20]. Sufficiently large fluctuations of these interaction matrix elements can explain the absence of bimodality in the peak spacing distribution 20, 21]. The variance of these fluctuations is determined by the spatial correlations of the single-particle wave functions. In a diffusive dot these correlations have been derived to leading order in $1 / g_{T}$, and the variance of the matrix elements of the screened Coulomb interaction was shown to behave as $\Delta^{2} / g_{T}^{2}[17,22]$, where $\Delta$ is the mean level spacing of the single-particle spectrum. However, dots studied in the experiments are usually ballistic. Wave function correlations in ballistic quantum dots are much less understood. Berry's conjecture [23] regarding the Gaussian nature of wave function fluctuations in chaotic systems provides the leading order behavior of the correlations at short distances, but finite-size contributions at distances that are comparable to the size of the dot can have important effects on the matrix element fluctuations.

An additional contribution to the peak spacing fluctuations originates in surface charge effects [17]. In a finite-size system, screening leads to the accumulation 
of charge on the surface of the dot. The confining onebody potential is then modified upon the addition of an electron to the dot. In a diffusive dot, the variance of one-body matrix elements behaves as $\Delta^{2} / g_{T}$.

Another interesting phenomenon, in which interaction matrix element fluctuations play an important role is spectral scrambling as electrons are added to a chaotic or diffusive dot [24, 25]. Both the two-body interaction and one-body surface charge effects are responsible for scrambling.

Here we investigate fluctuations of the two-body interaction matrix elements and of the surface charge potential matrix elements in ballistic dots. We use a normalized version of Berry's random wave model to derive analytically leading-order contributions for an arbitrary dot geometry, and compare with exact numerical simulations.

The outline of this paper is as follows. In Sec. III we review the random wave model for chaotic billiards. The spatial correlator of wave function intensity obtained from this model is geometry-independent but not consistent with the normalization requirement of the wave functions [26]. We compute normalization corrections to the correlator in Sec. III] The variances of diagonal, double-diagonal, and off-diagonal two-body interaction matrix elements are calculated in Sec. IV and are all found to be strongly affected by the normalization correction. As a result, ratios of these variances are shown to remain far from their asymptotic $k L \rightarrow \infty$ values for the range $30 \leq k L \leq 70$ that is relevant to experiments. In Sec. IV C we study the covariance of interaction matrix elements, relevant for understanding spectral scrambling when several electrons are added to the dot [24], and supplement the full random wave analysis with a schematic random matrix model. One-body matrix element variances are treated in Sec. V In all of these studies we compare numerical results of the random wave model with leading-order analytical estimates. In Sec. VI] we study the interaction matrix element distributions and show that, in the experimentally accessible range of $k L$, they deviate significantly from the Gaussian limit implied by the central limit theorem. Finally, in Sec. VII we address additional implications of the present work, including the necessity to supplement the normalized random wave model with dynamical effects for quantitative comparison with experiment.

\section{RANDOM WAVE MODEL}

In a chaotic system without symmetries, a typical classical trajectory will uniformly explore an entire energy hypersurface in phase space. A well-established and extensively tested conjecture by Berry [23] holds that in the quantization of such a system, a typical wave function will spread uniformly over an energy hypersurface, up to the inevitable Gaussian random amplitude fluctuations that arise whenever a random vector is expanded in a generic basis. In a semiclassical picture, the Gaussian fluctuations are the result of exponentially many wave fronts visiting any given region of phase space with quasirandom phases.

For a two-dimensional billiard system, the random wave model implies that a typical chaotic wave function may be written locally as a random superposition of plane waves at fixed energy $\hbar^{2} k^{2} / 2 m$ :

$$
\psi(\vec{r})=\psi(r, \theta)=\int_{0}^{2 \pi} d \phi A(\phi) e^{i k r \cos (\theta-\phi)},
$$

where $A(\phi)$ is distributed as a $\delta$-correlated Gaussian random variable: $\overline{A(\phi)}=0$ and $\overline{A^{*}(\phi) A\left(\phi^{\prime}\right)}=\frac{1}{2 \pi V} \delta\left(\phi-\phi^{\prime}\right)$. The normalization is fixed by $\overline{|\psi(\vec{r})|^{2}}=1 / V$, where $V$ is the area of the billiard. Equivalently, the wave function may be expanded in circular waves with good angular momentum $\mu$ :

$$
\psi(\vec{r})=\sum_{\mu=-\infty}^{\infty} B_{\mu} \phi_{\mu}(\vec{r}),
$$

where

$$
\phi_{\mu}(\vec{r}) \equiv J_{\mu}(k r) e^{i \mu \theta} .
$$

The discrete variables $B_{\mu}(\mu=0, \pm 1, \pm 2, \ldots)$ are taken to be uncorrelated Gaussian random variables with

$$
\overline{B_{\mu}}=0 \quad \overline{B_{\mu}^{*} B_{\mu^{\prime}}}=\frac{1}{V} \delta_{\mu \mu^{\prime}} .
$$

The normalization integral of a wave function (2) is given by

$$
\frac{1}{V} \int_{V} d \vec{r}|\psi(\vec{r})|^{2}=\sum_{\mu \mu^{\prime}} B_{\mu}^{*} A_{\mu \mu^{\prime}} B_{\mu^{\prime}},
$$

where $A_{\mu \mu^{\prime}}$ are the basis state overlaps

$$
A_{\mu \mu^{\prime}}=\frac{1}{V} \int_{V} d \vec{r} \phi_{\mu}^{*}(\vec{r}) \phi_{\mu^{\prime}}(\vec{r}) .
$$

Defining $\mathbf{B}$ to be a column vector with components $B_{\mu}$, the normalization integral of the wave function $\psi$ is simply the norm of $\mathbf{B}$ with the matrix $A$ playing the role of a metric

$$
\frac{1}{V} \int_{V} d \vec{r}|\psi(\vec{r})|^{2}=\mathbf{B}^{\dagger} A \mathbf{B}
$$

The random coefficients $B_{\mu}$ may be chosen to either satisfy or not satisfy the real wave function condition $B_{-\mu}=B_{\mu}^{*}$, corresponding to the absence or presence of an external magnetic field; these two situations are conventionally denoted by $\beta=1,2$ respectively.

Using the addition theorem for Bessel functions we have

$$
\sum_{\mu=-\infty}^{\infty} \phi_{\mu}^{*}(\vec{r}) \phi_{\mu}\left(\vec{r}^{\prime}\right)=J_{0}\left(k\left|\vec{r}-\vec{r}^{\prime}\right|\right)
$$


It follows from the completeness property (8) of the Bessel basis that

$$
\operatorname{tr} A=\sum_{\mu} A_{\mu \mu}=1
$$

We note that formally the model requires an infinite set of basis states; in practice the effective number $N_{\text {eff }}$ of basis states that have appreciable magnitude inside the area $V$ scales as $N_{\text {eff }} \sim k L \sim g_{T}$, where $L \equiv \sqrt{V}$ is a typical linear size of the billiard and $g_{T}$ is the ballistic Thouless conductance. A more accurate estimate for $N_{\text {eff }}$ can be obtained by considering a disk of radius $R=L / \sqrt{\pi}$. In a disk, the wave functions $\phi_{\mu}$ are orthogonal because of rotational symmetry and $A_{\mu \mu^{\prime}} \propto \delta_{\mu \mu^{\prime}}$. The effective dimension $N_{\text {eff }}$ is then obtained as the "participation number" of the exact $A_{\mu \mu}$ :

$$
N_{\mathrm{eff}}=\left[\sum_{\mu}\left|A_{\mu \mu}\right|^{2}\right]^{-1} \approx 1.85 k R \approx 1.04 k L
$$

The approximate completeness of a basis of $N_{\text {eff }} \sim g_{T}$ plane waves at a fixed energy $\hbar^{2} k^{2} / 2 m$ was confirmed in studies of a billiard system [27].

\section{INTENSITY CORRELATOR}

The random wave model of Eqs. (2) and (4) together with Eq. (8) leads to the amplitude correlator

$$
\overline{\psi^{*}(\vec{r}) \psi\left(\vec{r}^{\prime}\right)}=\frac{1}{V} J_{0}\left(k\left|\vec{r}-\vec{r}^{\prime}\right|\right)
$$

and intensity correlator

$$
\begin{aligned}
& \overline{|\psi(\vec{r})|^{2}\left|\psi\left(\vec{r}^{\prime}\right)\right|^{2}}-\overline{|\psi(\vec{r})|^{2}} \overline{\left|\psi\left(\vec{r}^{\prime}\right)\right|^{2}} \\
& \approx C\left(\vec{r}, \vec{r}^{\prime}\right)=\frac{1}{V^{2}} \frac{2}{\beta} J_{0}^{2}\left(k\left|\vec{r}-\vec{r}^{\prime}\right|\right) .
\end{aligned}
$$

Eq. (12) is obtained from Eq. (11) by contracting $\psi(\vec{r})$ with $\psi^{*}\left(\vec{r}^{\prime}\right)$ and $\psi^{*}(\vec{r})$ with $\psi\left(\vec{r}^{\prime}\right)$ and noting that there are two equivalent ways of performing the contraction when $\psi=\psi^{*}$ (i.e., for $\beta=1$ ).

Similar correlators can be derived starting from the plane-wave expansion (11). The intensity correlator (12) is valid to leading order in $|\vec{r}-\vec{r}| / L$, but becomes problematic when applied to to all $\vec{r}, \vec{r}^{\prime}$ in the finite area $V$. Indeed wave function normalization requires the correlator to vanish on average [22],

$$
\int_{V} d \vec{r}\left[\overline{|\psi(\vec{r})|^{2}\left|\psi\left(\vec{r}^{\prime}\right)\right|^{2}}-\overline{|\psi(\vec{r})|^{2}} \overline{\left|\psi\left(\vec{r}^{\prime}\right)\right|^{2}}\right]=0
$$

and similarly $\int_{V} d \vec{r}^{\prime}[\ldots]=0$. However, the random wave intensity correlator $C\left(\vec{r}, \vec{r}^{\prime}\right)$ in (12) is non-negative everywhere and does not satisfy the condition (13). The reason for this failure is that in the random wave model normalization is satisfied only on average, i.e., $\overline{\int_{V} d \vec{r}|\psi(\vec{r})|^{2}}=$ $\operatorname{tr} A=1$, but not for each individual wave function.
This deficiency can be corrected by introducing the normalized random wave model, in which each "random" wave function (2) is normalized in area $V$, i.e.,

$$
\psi^{\mathrm{norm}}(\vec{r})=\sum_{\mu} B_{\mu}^{\mathrm{norm}} J_{\mu}(k r) e^{i \mu \theta}
$$

with $B_{\mu}^{\text {norm }}=B_{\mu} / \sqrt{V \mathbf{B}^{\dagger} A \mathbf{B}}$. The normalized random wave model is easy to implement numerically by normalizing each random wave. Analytically, the intensity correlator of this model can be written as

$$
C^{\text {norm }}\left(\vec{r}, \vec{r}^{\prime}\right)=\overline{\left(\left|\psi^{\text {norm }}(\vec{r})\right|^{2}-\frac{1}{V}\right)\left(\left|\psi^{\text {norm }}\left(\vec{r}^{\prime}\right)\right|^{2}-\frac{1}{V}\right)} .
$$

This is similar to the situation in random matrix theory (RMT), where the naive guess $\overline{\left|a_{i}\right|^{2}\left|a_{j}\right|^{2}}-\overline{\left|a_{i}\right|^{2}} \overline{\left|a_{j}\right|^{2}}=$ $\frac{1}{N^{2}} \delta_{i j}$ for $\beta=2\left(a_{i}\right.$ and $a_{j}$ are two components of an RMT eigenvector of length $N$ with normalization $\left.\sum_{i}\left|a_{i}\right|^{2}=1\right)$ must be replaced by the exact expression $\frac{1}{N(N+1)}\left[\delta_{i j}-\frac{1}{N}\right]$ to obtain correct normalization for finite $N$. In our case, however, the normalized intensity correlator (15) depends on both positions $\vec{r}$ and $\vec{r}^{\prime}$ as well as the system geometry.

We define at a spatial point $\vec{r}$ an excess wave-function intensity by $u(\vec{r})=|\psi(\vec{r})|^{2}-\frac{1}{V}$ and similarly an excess normalized wave-function intensity $u^{\operatorname{norm}}(\vec{r})=$ $\left|\psi^{\text {norm }}(\vec{r})\right|^{2}-\frac{1}{V}$. We then have

$$
=\frac{C^{\operatorname{norm}}\left(\vec{r}, \vec{r}^{\prime}\right)=\overline{u^{\operatorname{norm}}(\vec{r}) u^{\operatorname{norm}}\left(\vec{r}^{\prime}\right)}}{\left(\frac{\frac{1}{V}+u(\vec{r})}{1+\int_{V} d \vec{r}_{a} u\left(\vec{r}_{a}\right)}-\frac{1}{V}\right)\left(\frac{\frac{1}{V}+u\left(\vec{r}^{\prime}\right)}{1+\int_{V} d \vec{r}_{b} u\left(\vec{r}_{b}\right)}-\frac{1}{V}\right)} .
$$

Eq. (16) is exact but unwieldy. In the semiclassical limit of large $k L$, a given superposition $\psi$ of random waves will be almost normalized, i.e., $\int_{V} d \vec{r}|\psi(\vec{r})|^{2}-1=$ $\int_{V} d \vec{r} u(\vec{r})=O\left((k L)^{-1 / 2}\right)$. Thus, to leading order in $1 / k L$, we may expand Eq. (16) to obtain

$$
\overline{\left(u(\vec{r})-\frac{1}{V} \int_{V} d \vec{r}_{a} u\left(\vec{r}_{a}\right)\right)\left(u\left(\vec{r}^{\prime}\right)-\frac{1}{V} \int_{V} d \vec{r}_{b} u\left(\vec{r}_{b}\right)\right)+\cdots,}
$$

where we have omitted all terms involving three-point and higher-order correlations of the excess intensity $u$. Eq. (16) can be simplified to obtain

$$
C^{\mathrm{norm}}\left(\vec{r}, \vec{r}^{\prime}\right)=\tilde{C}\left(\vec{r}, \vec{r}^{\prime}\right)+O\left(\frac{1}{(k L)^{3 / 2}}\right),
$$

where

$$
\begin{aligned}
\tilde{C}\left(\vec{r}, \vec{r}^{\prime}\right) & =C\left(\vec{r}, \vec{r}^{\prime}\right)-\frac{1}{V} \int_{V} d \vec{r}_{a} C\left(\vec{r}, \vec{r}_{a}\right) \\
& -\frac{1}{V} \int_{V} d \vec{r}_{a} C\left(\vec{r}_{a}, \vec{r}^{\prime}\right) \\
& +\frac{1}{V^{2}} \int_{V} \int_{V} d \vec{r}_{a} d \vec{r}_{b} C\left(\vec{r}_{a}, \vec{r}_{b}\right) .
\end{aligned}
$$


The leading-order normalized correlator $\tilde{C}\left(\vec{r}, \vec{r}^{\prime}\right)$ was derived in Ref. 26] by adding a weak smooth disorder and using the non-linear supersymmetric sigma model. More recently, the same leading correction has been obtained [28] starting from a density matrix and the principle of maximum entropy [29]. Here we we have shown that this form can be obtained quite generally by applying a perturbative approach to the unnormalized correlator $C\left(\vec{r}, \vec{r}^{\prime}\right)$, assuming only that correlations are weak in the limit of large system size $k L$.

We note, however, that $\tilde{C}\left(\vec{r}, \vec{r}^{\prime}\right)$ is merely a leading order approximation in $1 / k L$ to the true normalized random wave correlator $C^{\text {norm }}\left(\vec{r}, \vec{r}^{\prime}\right)$, as it involves only terms depending on the unnormalized two-point correlator $C$. The complete expression (16) for the normalized two-point correlator $C^{\text {norm }}\left(\vec{r}, \vec{r}^{\prime}\right)$ involves all unnormalized $n$-point correlators $\overline{u\left(\vec{r}_{1}\right) \cdots u\left(\vec{r}_{n}\right)}$. In particular, the unnormalized three-point correlator gives rise to the $O\left((k L)^{-3 / 2}\right)$ correction in Eq. (18). In principle, such higher-order corrections to Eq. (18) may be computed systematically, but in practice their effect on matrix element variances is small, as we will confirm below. The $n$-point correlators $\overline{u\left(\vec{r}_{1}\right) \cdots u\left(\vec{r}_{n}\right)}$ for $n \geq 3$ will be important when we discuss the deviation of the matrix element distribution from a Gaussian (see Sec. VI). We also note that our definition of $C^{\text {norm }}\left(\vec{r}, \vec{r}^{\prime}\right)$ and the approach of Refs. [28, 29] may produce different corrections to $\tilde{C}\left(\vec{r}, \vec{r}^{\prime}\right)$ at higher orders in a $1 / k L$ expansion. Although the problem of constructing the "best" random wave ensemble that exactly satisfies normalization constraints is an interesting one in its own right, the effect of these higher-order ambiguities on matrix element statistics is negligible compared to dynamical contributions in real chaotic systems.

In contrast to the unnormalized correlator $C\left(\vec{r}, \vec{r}^{\prime}\right)$, the normalized correlator $\tilde{C}\left(\vec{r}, \vec{r}^{\prime}\right)$ satisfies

$$
\int_{V} d \vec{r}^{\prime} \tilde{C}\left(\vec{r}, \vec{r}^{\prime}\right)=0
$$

implying the normalization of each individual wave function $\int_{V} d \vec{r}\left|\psi_{\alpha}(\vec{r})\right|^{2}=1$. To show this, we define $\mathcal{N}_{\alpha}=$ $\int_{V} d \vec{r}\left|\psi_{\alpha}(\vec{r})\right|^{2}$. We note that $\overline{\mathcal{N}}=1$ by construction, and calculate the variance in wave function normalization:

$$
\begin{aligned}
\overline{\mathcal{N}^{2}}-\overline{\mathcal{N}}^{2} & =\int_{V} \int_{V} d \vec{r} d \vec{r}^{\prime} \overline{|\psi(\vec{r})|^{2}\left|\psi\left(\vec{r}^{\prime}\right)\right|^{2}}-1 \\
& =\int_{V} \int_{V} d \vec{r} d \vec{r}^{\prime}\left(\frac{1}{V^{2}}+\tilde{C}\left(\vec{r}, \vec{r}^{\prime}\right)\right)-1 \\
& =\int_{V} \int_{V} d \vec{r} d \vec{r}^{\prime} \tilde{C}\left(\vec{r}, \vec{r}^{\prime}\right) .
\end{aligned}
$$

Eq. (20) implies that the variance of $\mathcal{N}$ vanishes, and since the average wave function is normalized to unity, every wave function in the ensemble must be normalized to unity.

A more schematic random matrix approximation is obtained if the exact metric $A_{\mu \mu^{\prime}}$ is assumed to be diagonal and the diagonal components are replaced by

$$
A_{\mu \mu}= \begin{cases}1 / N_{\mathrm{eff}} & \text { for }|\mu|<N_{\mathrm{eff}} / 2 \\ 0 & \text { for }|\mu| \geq N_{\mathrm{eff}} / 2\end{cases}
$$

The distribution (22) satisfies $\sum_{\mu} A_{\mu \mu}=1$ and $\sum_{\mu}\left|A_{\mu \mu}\right|^{2}=1 / N_{\text {eff }}$ and therefore reproduces correctly the first two moments (9) and (10) of the exact $A_{\mu \mu}$ distribution. In the approximation (22), the normalization of the wave function reads $\mathbf{B}^{\dagger} \mathbf{B}=N_{\text {eff }} / V$ [see

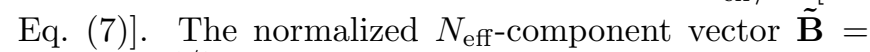
$\left(N_{\text {eff }} / V\right)^{-1 / 2} \mathbf{B}$ can then be viewed as an eigenvector of a Gaussian random matrix of the corresponding symmetry class $\beta$.

In a disk of radius $R$, asymptotic expressions may be used to verify that the exact self-overlaps of the Bessel functions approach a constant $A_{\mu \mu} \approx 2 / \pi k R$ for $\mu \ll N_{\text {eff }} \sim k R$ and fall off exponentially as $A_{\mu \mu} \approx$ $\frac{1}{2 \pi \mu^{2}}\left(\frac{e k R}{2 \mu}\right)^{2 \mu}$ for $\mu \gg N_{\text {eff }} \sim k R$. In Fig. 1] we show the exact random-wave metric for a disk with $k R=40$ and $k R=100$, as well as the schematic random matrix approximation (22).

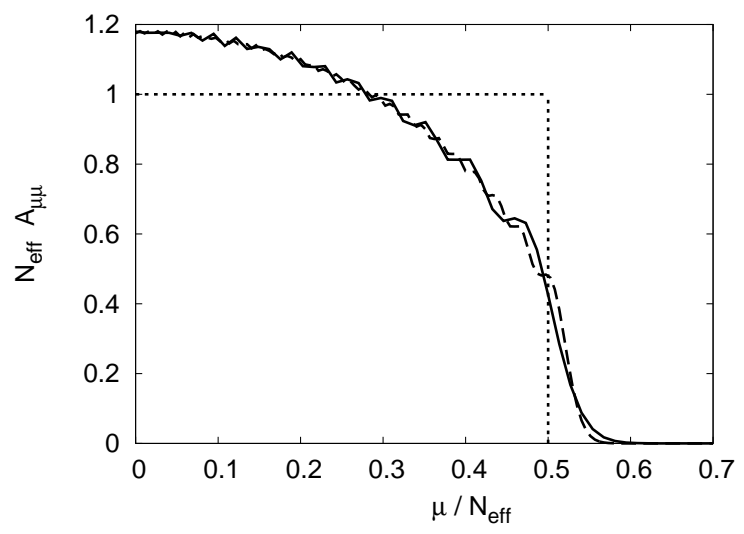

FIG. 1: The exact Bessel function self-overlaps $A_{\mu \mu}$ defined by Eq. (6) are computed for a disk of radius $R$, and scaled by the effective Hilbert space dimension $N_{\text {eff }}=1.85 k R$. The solid curve indicates $k R=40$, while the dashed curve corresponds to $k R=100$. The dotted rectangle represents the schematic random matrix approximation, where all $A_{\mu \mu}$ are taken to be constant for $-N_{\text {eff }} / 2<\mu<N_{\text {eff }} / 2$ and 0 otherwise. Quantities plotted in this and all subsequent figures are dimensionless.

In the following sections we use the random wave model to estimate the variances of various matrix elements of the screened Coulomb interaction. Normalized random waves can be generated numerically to calculate the spatial wave function correlator and the corresponding variances, which is equivalent to using the exact normalized intensity correlator $C^{\text {norm }}$. Analytical expressions can be obtained as follows. (i) The asymptotic behavior of the unnormalized random wave correlator $C\left(\vec{r}, \vec{r}^{\prime}\right)$ in Eq. (12) will be sufficient to produce analytically the leading geometry-independent $O\left(\ln k L /(k L)^{2}\right)$ 
behavior of two-body interaction matrix element variances. (ii) A direct integration of the normalized correlator $\tilde{C}\left(\vec{r}, \vec{r}^{\prime}\right)$ in Eq. (19) produces geometry-dependent $O\left(1 /(k L)^{2}\right)$ terms for the above matrix elements. This correlator can also be used to calculate to leading order in $k L$ the size of one-body surface charge matrix element fluctuations.

\section{TWO-BODY INTERACTION MATRIX ELEMENTS}

Here we model the screened two-body Coulomb interaction in $2 \mathrm{D}$ quantum dots as a contact interaction $v\left(\vec{r}, \vec{r}^{\prime}\right)=\Delta V \delta\left(\vec{r}-\vec{r}^{\prime}\right)$, where the single-particle mean level spacing $\Delta$ serves to set the energy scale [30, 31].

\section{A. Fluctuation of diagonal matrix elements $v_{\alpha \beta}$}

We first discuss the variance of a diagonal two-body matrix element $v_{\alpha \beta} \equiv v_{\alpha \beta ; \alpha \beta}$. In the contact interaction model

$$
v_{\alpha \beta}=\Delta V \int_{V} d \vec{r}\left|\psi_{\alpha}(\vec{r})\right|^{2}\left|\psi_{\beta}(\vec{r})\right|^{2} .
$$

To leading order in $g_{T} \sim k L$, the dominant contribution to the variance arises from correlations between the intensities of a single wave function at different points [24]:

$$
\overline{\delta v_{\alpha \beta}^{2}}=\Delta^{2} V^{2} \int_{V} \int_{V} d \vec{r} d \vec{r}^{\prime} \tilde{C}^{2}\left(\vec{r}, \vec{r}^{\prime}\right)+\cdots
$$

Since this leading-order effect comes from correlations within a single wave function, the only restriction on the energy difference $E_{\alpha}-E_{\beta}$ between the two random wave functions is $\left|E_{\alpha}-E_{\beta}\right| \ll E_{\alpha}$, or equivalently $\left|k_{\alpha}-k_{\beta}\right| \ll k_{\alpha}$, allowing the variance to be a function of a single parameter $k L$. The subleading $O\left(1 /(k L)^{3}\right)$ terms omitted in Eq. (24) involve the two-eigenstate intensity correlator

$$
\tilde{C}_{2}\left(\vec{r}, \vec{r}^{\prime}\right)=\overline{\left|\psi_{\alpha}(\vec{r})\right|^{2}\left|\psi_{\beta}\left(\vec{r}^{\prime}\right)\right|^{2}}-\overline{\left|\psi_{\alpha}(\vec{r})\right|^{2}} \overline{\left|\psi_{\beta}\left(\vec{r}^{\prime}\right)\right|^{2}} .
$$

The correlator (25) produces the leading effect for the covariance and will be discussed in detail in Section IV C

The leading-order contribution to Eq. (24) is obtained by using the unnormalized correlator $C\left(\vec{r}, \vec{r}^{\prime}\right)$ instead of $\tilde{C}\left(\vec{r}, \vec{r}^{\prime}\right)$. Changing integration variables in Eq. (24) to $\vec{R}=\left(\vec{r}+\vec{r}^{\prime}\right) / 2$ and $\vec{\rho}=\vec{r}-\vec{r}^{\prime}$, inserting the unnormalized correlator (12), which depends only on $k \rho$, and substituting the asymptotic form $J_{0}^{2}(k \rho)=\frac{2}{\pi k \rho} \cos ^{2}(k \rho-\pi / 4)+$ $O\left(1 /(k \rho)^{2}\right)$, we obtain

$$
\begin{aligned}
\overline{\delta v_{\alpha \beta}^{2}} & \approx \frac{\Delta^{2}}{V}\left(\frac{2}{\beta}\right)^{2} \int_{1 / k}^{L} d \rho(2 \pi \rho) \frac{4}{\pi^{2} k^{2} \rho^{2}} \cos ^{4}(k \rho-\pi / 4) \\
& =\Delta^{2} \frac{3}{\pi}\left(\frac{2}{\beta}\right)^{2} \frac{\ln k L}{(k L)^{2}}
\end{aligned}
$$

where in the last line we have used $\overline{\cos ^{4} k \rho}=3 / 8$.

In Eq. (26) we have not properly included the short distance contribution from $\rho \sim 1 / k$ and the shapedependent long-distance contribution from $\rho \sim L$. Both of these contributions to Eq. (24) scale as $1 / k^{2}$; in the first case because $\rho \sim 1 / k$ defines an $O\left(1 / k^{2}\right)$ volume in $\vec{\rho}$-space, and in the second case because $J_{0}^{4}(k \rho) \sim$ $1 /(k L)^{2}$ for $\rho \sim L$. To obtain the correct result at this subleading order we need to use the normalized correlator of Eq. (19). We then obtain

$$
\begin{aligned}
\overline{\delta v_{\alpha \beta}^{2}} & =\Delta^{2} V^{2} \int_{V} \int_{V} d \vec{r} d \vec{r}^{\prime} \tilde{C}^{2}\left(\vec{r}, \vec{r}^{\prime}\right)+O\left(\frac{\Delta^{2}}{(k L)^{3}}\right) \\
& =\Delta^{2} \frac{3}{\pi}\left(\frac{2}{\beta}\right)^{2} \frac{\ln k L+b_{g}}{(k L)^{2}}+O\left(\frac{\Delta^{2}}{(k L)^{3}}\right) .
\end{aligned}
$$

The leading $\ln k L /(k L)^{2}$ term, discussed in Ref. [31], depends only on the symmetry class, while the shapedependent coefficient $b_{g}$ can be easily evaluated by numerical integration of Eq. (27).

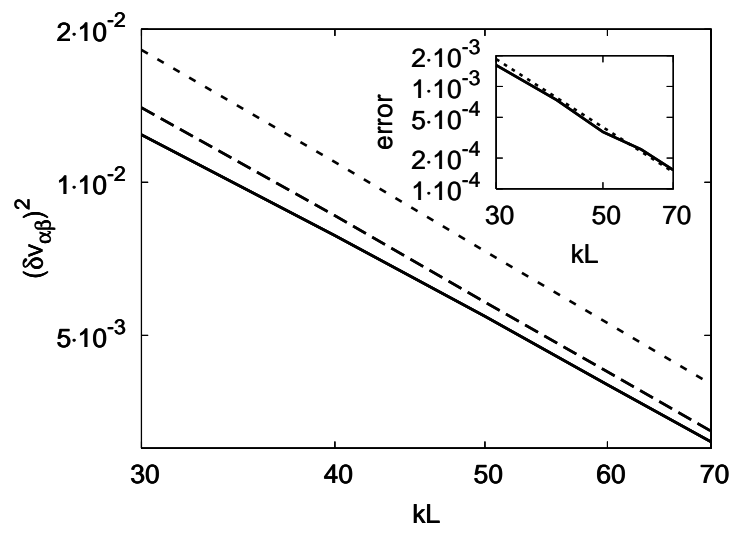

FIG. 2: The variance of the two-body matrix element $v_{\alpha \beta}$ versus $k L$ in the real $(\beta=1)$ random wave model: (a) the solid curve is the result of exact numerical simulations; (b) the long-dashed line is the result of integrating the square of the normalized correlator $\tilde{C}^{2}\left(\vec{r}, \vec{r}^{\prime}\right)$, as in Eq. (27); (c) the shortdashed line is the result of using the unnormalized correlator $C^{2}\left(\vec{r}, \vec{r}^{\prime}\right)$. In the inset, the solid line is the difference between the full result (a) and the approximation (b); the dotted line indicates that omitted terms scale as $\frac{1}{(k L)^{3}}$. Here and in all following figures, the level spacing $\Delta$, which sets the overall energy scale, is set to unity.

In Fig. 2] we show the results for a disk geometry in the range $30 \leq k L \leq 70$, corresponding roughly to the parameter range relevant for experiments $(\sim 150-800$ electrons in the dot). The numerical simulation of $\overline{\delta v_{\alpha \beta}^{2}}$ (solid line) is compared with the leading result of Eq. (27) (long-dashed line). For the disk, we find $b_{g}=-0.1$. The difference between the full numerical calculation and the integral of the normalized correlator is plotted in the inset, and is seen to be in good agreement with the $1 /(k L)^{3}$ scaling of Eq. (27). If the unnormalized correlator (12) is 
used in (27) we find (for the disk geometry) $b_{g}^{\text {unnorm }}=0.9$. The corresponding variance is shown as a short-dashed line in Fig. 2 ,

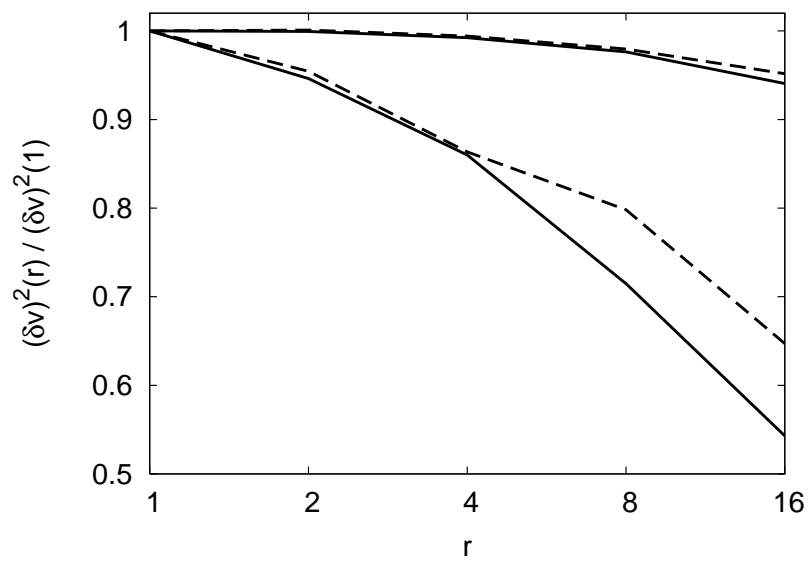

FIG. 3: The change in the $v_{\alpha \beta}$ variance (upper two curves) and the variance of the one-body matrix element $v_{\alpha}$ (lower two curves, see Sec. $\mathrm{V}$ for normalized real random waves are plotted as functions of the aspect ratio $r=a / b$ of the elliptical geometry. Here $k L=k \sqrt{\pi a b}$ is kept fixed at 30 (solid curves) and 70 (dashed curves).

The dependence of the $v_{\alpha \beta}$ fluctuations on the dot's geometry is demonstrated in the upper two curves Fig. 3 . Here we plot the result of Eq. (27) for elliptical shapes as a function of the aspect ratio $r$ between the major and minor axes, while keeping the area and the wave vector $k$ fixed. We find that as the aspect ratio changes by a (physically unrealistic) factor of 16, the shape-dependent parameter $b_{g}$ only changes from -0.1 to -0.3 , resulting in a change of only $\sim 5-6 \%$ in the $v_{\alpha \beta}$ variance for the experimental range of $k L$. Similar results are found if elliptical shapes are replaced by rectangles or other geometries. Thus, for all practical purposes, shape effects on the $v_{\alpha \beta}$ variance can be ignored (at least within the normalized random wave model).

At this subleading order, we must in principle also take into account the energy difference $E_{\alpha}-E_{\beta}$, if this energy difference is larger than the ballistic Thouless energy $g_{T} \Delta$ (but still small compared with the average $\left(E_{\alpha}+E_{\beta}\right) / 2$ ). Then $1 / L \ll \delta k=k_{\alpha}-k_{\beta} \ll k=\left(k_{\alpha}+k_{\beta}\right) / 2$, and the $\cos ^{4}(k \rho-\pi / 4)$ factor in Eq. (26) must be replaced by $\cos ^{2}\left(k_{\alpha} \rho-\pi / 4\right) \cos ^{2}\left(k_{\beta} \rho-\pi / 4\right)$, which has the average value $3 / 8$ only for $1 / k \ll \rho \ll 1 / \delta k$ and is reduced to an average of $1 / 4$ for $1 / \delta k \ll \rho \ll L$. After performing the integration over $\rho$ in Eq. (26), we find that $\ln k L$ in the final result must be replaced with $\ln k L-(1 / 3) \ln \delta k L$. Assuming $\delta k L$ remains large but fixed while $k L \rightarrow \infty$, this corresponds merely to a modification of the geometrydependent coefficient in Eq. (28): $b_{g} \rightarrow b_{g}-(1 / 3) \ln \delta k L$ for $\delta k L \gg 1$. In practice, for reasonable energy windows, e.g., $\delta k L \sim 5$, the consequent reduction in the variance is at most $10 \%$ and may be safely ignored compared to the much larger dynamical effects present in real chaotic systems.

\section{B. Fluctuation of $v_{\alpha \alpha}$ and $v_{\alpha \beta \gamma \delta}$}

The preceding section studied fluctuations in the intensity overlap $v_{\alpha \beta}$ between two random wave functions $\psi_{\alpha}$ and $\psi_{\beta}$. Similar techniques may be applied to the self-overlap of a single wave function $v_{\alpha \alpha}=\Delta V \int_{V} d \vec{r}\left|\psi_{\alpha}(\vec{r})\right|^{4}$, also known as the inverse participation ratio in coordinate space, and to the "off-diagonal" four-wave-function overlap $v_{\alpha \beta \gamma \delta}=$ $\Delta V \int_{V} d \vec{r} \psi_{\alpha}^{*}(\vec{r}) \psi_{\beta}(\vec{r}) \psi_{\gamma}^{*}(\vec{r}) \psi_{\delta}(\vec{r})$.

To leading order, $O\left(\ln k L /(k L)^{2}\right)$, all the results arise from integrating the unnormalized correlator (12) and differ only by combinatorial factors. For example, the $\left(\frac{2}{\beta}\right)^{2}$ factor in Eq. (26) may be understood from the fact that there are four distinct ways to contract pairs of same-wave-function amplitudes between $\psi_{\alpha}^{*}(\vec{r}) \psi_{\alpha}(\vec{r}) \psi_{\beta}^{*}(\vec{r}) \psi_{\beta}(\vec{r})$ and $\psi_{\alpha}\left(\vec{r}^{\prime}\right) \psi_{\alpha}^{*}\left(\vec{r}^{\prime}\right) \psi_{\beta}\left(\vec{r}^{\prime}\right) \psi_{\beta}^{*}\left(\vec{r}^{\prime}\right)$ if the wave functions are real $(\beta=1)$, but only one way to perform this contraction if the wave functions are complex $(\beta=2)$. An analogous counting argument for the variance of $v_{\alpha \alpha}$ leads to

$$
\overline{\delta v_{\alpha \alpha}^{2}}=\Delta^{2} \frac{3}{\pi} c_{\beta} \frac{\ln k L+b_{g}^{\prime}}{(k L)^{2}}+O\left(\frac{\Delta^{2}}{(k L)^{3}}\right)
$$

with $c_{1}=24$ and $c_{2}=4$. We note that such combinatorial factors can also be derived from the invariance of the second moments of the matrix elements under a change of the single-particle basis 18.

Finally, if $\alpha, \beta, \gamma$, and $\delta$ are all different, there is only one way to perform the contraction in either the real or complex case, leading to

$$
\begin{aligned}
\overline{\delta v_{\alpha \beta \gamma \delta}^{2}} & =\Delta^{2} V^{2} \int_{V} \int_{V} d \vec{r} d \vec{r}^{\prime}\left[\overline{\psi^{*}(\vec{r}) \psi\left(\vec{r}^{\prime}\right)}\right]^{4} \\
& =\Delta^{2} \frac{3}{\pi} \frac{\ln k L+b_{g}^{\prime \prime}}{(k L)^{2}}+O\left(\frac{\Delta^{2}}{(k L)^{3}}\right)
\end{aligned}
$$

While the leading $\ln k L /(k L)^{2}$ behavior in Eqs. (29) nd (30) is identical to that for $\overline{\delta v_{\alpha \beta}^{2}}$ up to geometryindependent combinatorial factors, the coefficients of the subleading geometry-dependent $1 /(k L)^{2}$ terms are not so simply related, as indicated by $b_{g}^{\prime}, b_{g}^{\prime \prime} \neq b_{g}$ in the above expressions. That is because the normalization-related subtraction, which enters at $O\left(1 /(k L)^{2}\right)$, works differently for the three matrix elements. For example, the variance of $v_{\alpha \beta}$ involves the product of two normalized intensity correlators $\tilde{C}^{2}\left(\vec{r}, \vec{r}^{\prime}\right) \sim\left(J_{0}\left(k\left|\vec{r}-\vec{r}^{\prime}\right|\right)^{2}-\cdots\right)^{2}$. On the other hand, the variance of $v_{\alpha \beta \gamma \delta}$ involves the product of four amplitude correlators (i.e., ${\overrightarrow{\psi^{*}(\vec{r}) \psi\left(\vec{r}^{\prime}\right)}}^{4} \sim$ $\left.J_{0}\left(k\left|\vec{r}-\vec{r}^{\prime}\right|\right)^{4}\right)$, which do not require subtraction. As indicated earlier, the absence of subtraction in the integral results in $b_{g}^{\prime \prime}=0.9$ for a disk geometry, in contrast with 
$b_{g}=-0.1$. For $v_{\alpha \alpha}$ fluctuations, we find $b_{g}^{\prime}=-2.25$ for a disk [32]. Thus, although $\overline{\delta v_{\alpha \alpha}^{2}}$ and $\overline{\delta v_{\alpha \beta \gamma \delta}^{2}}$ are related to $\overline{\delta v_{\alpha \beta}^{2}}$ by geometry-independent universal factors in the $k L \rightarrow \infty$ limit, the convergence to this universal limit is logarithmically slow. Specifically, in the presence of time-reversal symmetry $(\beta=1)$,

$$
\begin{gathered}
\overline{\delta v_{\alpha \alpha}^{2}} / \overline{\delta v_{\alpha \beta}^{2}}=6+\frac{b_{g}^{\prime}-b_{g}}{\ln k L}+\cdots \\
\overline{\delta v_{\alpha \beta}^{2}} / \overline{\delta v_{\alpha \beta \gamma \delta}^{2}}=4+\frac{b_{g}-b_{g}^{\prime \prime}}{\ln k L}+\cdots,
\end{gathered}
$$

to leading order in $1 / \ln k L$.

Fig. 4 shows these ratios versus $k L$ in the physical range of interest. Note in particular the very slow convergence of Eq. (31) to the asymptotic value of 6 because of the large difference between the coefficients of the subleading terms $b_{g}^{\prime}-b_{g}=-2.15$.

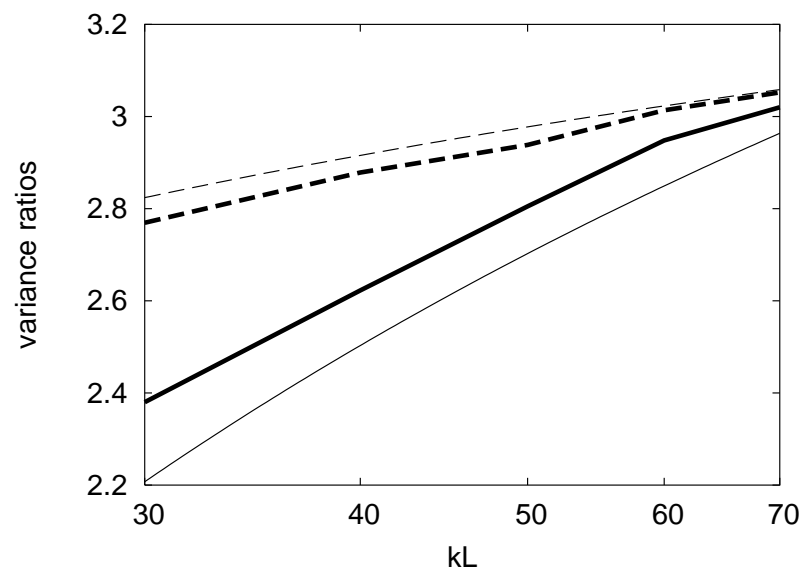

FIG. 4: Ratios of matrix element variances are plotted for real random waves inside a disk as functions of $k L: \overline{\delta v_{\alpha \alpha}^{2}} / \overline{\delta v_{\alpha \beta}^{2}}$ (thick solid line) and $\overline{\delta v_{\alpha \beta}^{2}} / \overline{\delta v_{\alpha \beta \gamma \delta}^{2}}$ (thick dashed line). For comparison, the leading-order analytic results of Eqs. (31) and (32) are shown as thin solid and dashed lines, respectively.

\section{Matrix element covariance $\overline{\delta v_{\alpha \beta} \delta v_{\alpha \gamma}}$}

Another quantity of interest is the covariance $\overline{\delta v_{\alpha \beta} \delta v_{\alpha \gamma}}$, where $\psi_{\alpha}, \psi_{\beta}$, and $\psi_{\gamma}$ are three distinct wave functions of a single dynamical system. Such matrix element covariances are important in quantitative estimates of scrambling of the Hartree-Fock single-particle spectrum as electrons are added to the dot [24]. As in Eq. (24), the leading contribution for large $k L$ can be written as a double integral of a product of two intensity correlators:

$$
\overline{\delta v_{\alpha \beta} \delta v_{\alpha \gamma}} \approx \int_{V} \int_{V} d \vec{r} d \vec{r}^{\prime} \tilde{C}\left(\vec{r}, \vec{r}^{\prime}\right) \tilde{C}_{2}\left(\vec{r}, \vec{r}^{\prime}\right),
$$

where $\tilde{C}\left(\tilde{r}, \vec{r}^{\prime}\right)$ is the intensity correlator for wave function $\psi_{\alpha}$ and $\tilde{C}_{2}\left(\vec{r}, \vec{r}^{\prime}\right)$ is the intensity correlator (25) between two distinct wave functions $\psi_{\beta}$ and $\psi_{\gamma}$. In a diffusive dot, expression (33) leads to a covariance $\propto \Delta^{2} / g_{T}^{3}$, where $g_{T}$ is the diffusive Thouless conductance [24].

We proceed to evaluate $\tilde{C}_{2}$ in the normalized random wave approximation. Clearly, if $\psi_{\beta}, \psi_{\gamma}$ are chosen to be independent random wave functions, the correlator vanishes by construction. Therefore, we need to impose the orthogonality condition

$$
\frac{1}{V} \int_{V} d \vec{r} \psi_{\beta}^{*}(\vec{r}) \psi_{\gamma}(\vec{r})=\mathbf{B}_{\beta}^{\dagger} A \mathbf{B}_{\gamma}=0,
$$

which may be done through the Gram-Schmidt procedure. We begin by generating two independent random vectors $\psi_{\beta}$ and $\psi_{\gamma}^{0}$ at the same energy $\hbar^{2} k^{2} / 2 m: \psi_{\beta}(\vec{r})=\sum_{\mu=-\infty}^{\infty} B_{\beta \mu} J_{\mu}(k r) e^{i \mu \theta}$ and $\psi_{\gamma}^{0}(\vec{r})=$ $\sum_{\mu=-\infty}^{\infty} B_{\gamma \mu} J_{\mu}(k r) e^{i \mu \theta}$, project $\psi_{\gamma}^{0}$ onto the subspace orthogonal to $\psi_{\beta}$, and normalize the result to unit length to find $\psi_{\gamma}$ :

$$
\psi_{\gamma}(\vec{r})=\frac{\psi_{\gamma}^{0}(\vec{r})-\left\langle\psi_{\beta} \mid \psi_{\gamma}^{0}\right\rangle \psi_{\beta}(\vec{r})}{\sqrt{1-\left|\left\langle\psi_{\beta} \mid \psi_{\gamma}^{0}\right\rangle\right|^{2}}} .
$$

Using the basis states $\phi_{\mu}$ in Eq. (3), we find

$$
\begin{array}{r}
\tilde{C}_{2}\left(\vec{r}, \vec{r}^{\prime}\right)=\frac{1}{V^{2}} \frac{2}{\beta}\left[J_{0}^{2}\left(k\left|\vec{r}-\vec{r}^{\prime}\right|\right) \sum_{\mu \mu^{\prime}}\left|A_{\mu \mu^{\prime}}\right|^{2}\right. \\
\left.-2 J_{0}\left(k\left|\vec{r}-\vec{r}^{\prime}\right|\right) \sum_{\mu \mu^{\prime}} A_{\mu \mu^{\prime}} \phi_{\mu}(\vec{r}) \phi_{\mu^{\prime}}^{*}\left(\vec{r}^{\prime}\right)\right]+O\left(\frac{1}{k^{2}}\right) .
\end{array}
$$

Eq. (36) can be evaluated numerically for a given geometry in order to obtain the covariance of diagonal matrix elements $v_{\alpha \beta}$ via Eq. (33). However, we obtain insight into the qualitative behavior of the correlator (36) by using the schematic random matrix model (22) in a disk geometry. We find

$$
\tilde{C}_{2}\left(\vec{r}, \vec{r}^{\prime}\right) \approx-\frac{1}{V^{2}} \frac{2}{\beta} \frac{1}{N_{\mathrm{eff}}} J_{0}^{2}\left(k\left|\vec{r}-\vec{r}^{\prime}\right|\right)=-\frac{1}{N_{\mathrm{eff}}} \tilde{C}\left(\vec{r}, \vec{r}^{\prime}\right),
$$

where $N_{\text {eff }}$ is the effective dimension defined by (10). Substituting the random matrix model relation (37) in (33) and using Eq. (24), we have

$$
\overline{\delta v_{\alpha \beta} \delta v_{\alpha \gamma}} \approx-\frac{1}{N_{\mathrm{eff}}} \overline{\delta v_{\alpha \beta}^{2}} .
$$

This simple relation between the variance and covariance of the interaction matrix elements could also be obtained directly from an effective completeness condition (valid for any $\alpha$ )

$$
\sum_{\beta=1}^{N_{\text {eff }}} \delta v_{\alpha \beta}=0
$$


where $\delta v_{\alpha \beta}=v_{\alpha \beta}-\overline{v_{\alpha \beta}}$. Eq. (39) follows from the completeness of the eigenstate basis $\sum_{\beta}\left|\psi_{\beta}(\vec{r})\right|^{2}=c$ (where $c$ is a position-independent constant). From Eq. (39) we find $\sum_{\beta \gamma} \delta v_{\alpha \beta} \delta v_{\alpha \gamma}=0$ or, after taking an ensemble average

$$
\sum_{\beta \neq \gamma} \overline{\delta v_{\alpha \beta} \delta v_{\alpha \gamma}}=-\sum_{\beta} \overline{\left(\delta v_{\alpha \beta}\right)^{2}} .
$$

If we assume the various covariances and variances to be independent of the specific orbitals, we recover the RMT relation (38).

Substituting Eqs. (10) and (28) into Eq. (38), we obtain an analytic result for the covariance in a circular dot in the RMT approximation:

$\overline{\delta v_{\alpha \beta} \delta v_{\alpha \gamma}} \approx-\Delta^{2} \frac{3}{\pi} \frac{\sqrt{\pi}}{1.85}\left(\frac{2}{\beta}\right)^{2} \frac{\ln k L+b_{g}}{(k L)^{3}}+O\left(\frac{\Delta^{2}}{(k L)^{4}}\right)$.

For a non-circular dot, we can write

$$
\phi_{\mu}(\vec{r}) \phi_{\mu^{\prime}}^{*}\left(\vec{r}^{\prime}\right)=A_{\mu \mu^{\prime}}^{*} J_{0}\left(k\left|\vec{r}-\vec{r}^{\prime}\right|\right)+f_{\mu \mu^{\prime}}\left(\vec{r}, \vec{r}^{\prime}\right),
$$

where from Eq. (8) $\sum_{\mu} f_{\mu \mu}=0$ for arbitrary $\vec{r}, \vec{r}^{\prime}$, and from Eq. (6) $\int_{V} d \vec{r} f_{\mu \mu^{\prime}}(\vec{r}, \vec{r})=0$ for arbitrary $\mu, \mu^{\prime}$. Although we have no formal justification for neglecting $f$, if we do so we obtain

$$
\tilde{C}_{2}\left(\vec{r}, \vec{r}^{\prime}\right) \approx-\left(\sum_{\mu \mu^{\prime}}\left|A_{\mu \mu^{\prime}}\right|^{2}\right) \tilde{C}\left(\vec{r}, \vec{r}^{\prime}\right)=-\frac{\tilde{C}\left(\vec{r}, \vec{r}^{\prime}\right)}{N_{\mathrm{eff}}}
$$

with

$$
N_{\mathrm{eff}}=\left[\sum_{\mu \mu^{\prime}}\left|A_{\mu \mu^{\prime}}\right|^{2}\right]^{-1} .
$$

The definition (44) of the effective dimension generalizes the finite RMT result of Eqs. (37) and (38) to an arbitrary shape. For an ellipse with an aspect ratio $a / b=2$, $N_{\text {eff }}$ increases by a factor of 1.12 as compared with a circle of the same area; for an ellipse with an aspect ratio $a / b=4, N_{\text {eff }}$ increases by a factor of 1.39 .

In Fig. 5 we compare the covariance $\overline{\delta v_{\alpha \beta} \delta v_{\alpha \gamma}}$ calculated numerically in the normalized random wave model with the RMT expression (41). Here we have again used a disk geometry, but very similar results are obtained in other geometries, e.g., a stadium billiard [33]. We note the surprisingly good agreement between the full numerical result and the schematic RMT approximation.

The arguments leading to Eq. (43) generalize to the case where $\psi_{\beta}$ and $\psi_{\gamma}$ are orthogonal random waves at different energies, as long as the energy difference is classically small, $\delta k \equiv\left|k_{\beta}-k_{\gamma}\right| \ll \bar{k} \equiv\left(k_{\beta}+k_{\gamma}\right) / 2$. Eq. (43) for the correlator is unchanged, provided we use the generalized definition

$$
A_{\mu \mu^{\prime}}=\frac{1}{V} \int_{V} d \vec{r} J_{\mu}\left(k_{\beta} r\right) e^{-i \mu \theta} J_{\mu^{\prime}}\left(k_{\gamma} r\right) e^{i \mu^{\prime} \theta},
$$

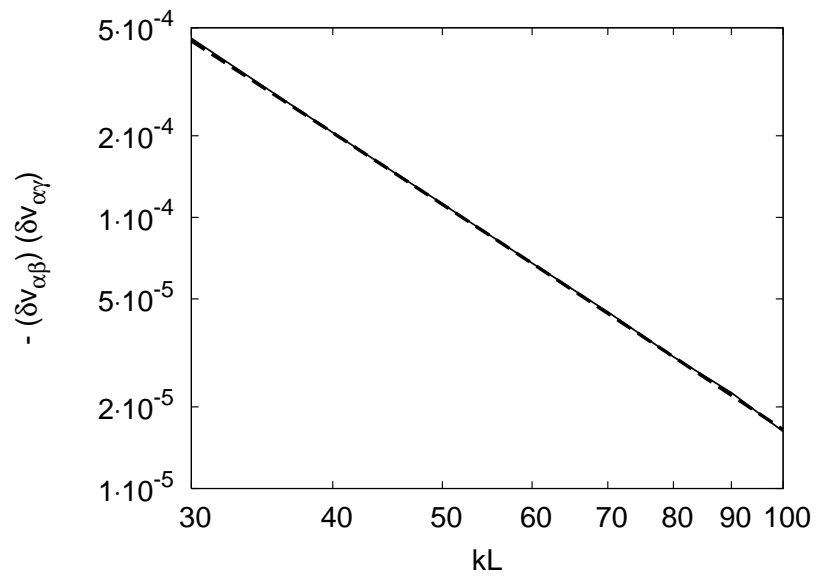

FIG. 5: The diagonal interaction matrix element covariance $\overline{\delta v_{\alpha \beta} \delta v_{\alpha \gamma}}$ (for wave functions $\psi_{\beta}$ and $\psi_{\gamma}$ at the same energy $\left.\hbar^{2} k^{2} / 2 m\right)$ is plotted versus $k L$ for real random waves inside a disk. The result obtained by substituting Eq. (36) into Eq. (33) and integrating numerically (solid line) is compared with the the analytic RMT approximation of Eq. (41) (dashed line).

and $\tilde{C}\left(\vec{r}, \vec{r}^{\prime}\right)$ is evaluated at $k=\bar{k}$. Note that $A_{\mu \mu^{\prime}}$, and thus $N_{\text {eff }}$ in Eq. (44), are now functions of two wave numbers $k_{\beta}$ and $k_{\gamma}$, or equivalently of $\bar{k}$ and $\delta k$. The final result for the covariance becomes

$$
\begin{aligned}
\overline{\delta v_{\alpha \beta} \delta v_{\alpha \gamma}} & \approx-\Delta^{2} \frac{3}{\pi} \frac{\bar{k} L}{N_{\mathrm{eff}}(\bar{k}, \delta k)}\left(\frac{2}{\beta}\right)^{2} \frac{\ln \bar{k} L+b_{g}}{(\bar{k} L)^{3}} \\
& +O\left(\frac{\Delta^{2}}{(\bar{k} L)^{4}}\right)
\end{aligned}
$$

where $N_{\text {eff }}$ depends implicitly on both wave vectors $k_{\beta}$ and $k_{\gamma}$ (or alternatively $\bar{k}$ and $\delta k$ ) through Eqs. (44) and (45).

The energy difference can be written as $E_{\beta}-E_{\gamma}=$ $2 \delta k \bar{k}\left(\hbar^{2} / 2 m\right) \sim(\delta k L) E_{T}$, where $E_{T} \sim \hbar /(L / v) \sim$ $\bar{k}\left(\hbar^{2} / m L\right)$ is the ballistic Thouless energy. When this energy difference is small compared with the Thouless energy, i.e., when the wave vector difference $\delta k$ is quantummechanically small $(\delta k L \ll 1)$, the ratio $N_{\text {eff }} / \bar{k} L$ reduces to a shape-dependent constant independent of $\bar{k}$. For a disk, this constant is $1.85 / \sqrt{\pi}$ [see Eq. (10)] and Eq. (46) reduces to Eq. (41).

On the other hand, when the energy difference is quantum mechanically large (i.e., large compared with the Thouless energy) but still classically small $(1 \ll \delta k L \ll$ $\bar{k} L)$, then the oscillatory functions $J_{\mu}\left(k_{\beta} r\right)$ and $J_{\mu^{\prime}}\left(k_{\gamma} r\right)$ go in and out of phase with each other $O(\delta k L)$ times between $r=0$ and $r \sim L$. The overlaps $A_{\mu \mu^{\prime}}$ as defined by Eq. (45) are therefore reduced by a factor $1 / \delta k L$ (as compared with the case $\delta k L \ll 1$ ), and $N_{\text {eff }}$ in Eq. (44) increases

$$
N_{\mathrm{eff}}(\delta k L) \sim(\delta k L)^{2} \cdot N_{\mathrm{eff}}(\delta k L=0) \sim(\delta k L)^{2} \cdot \bar{k} L .
$$


$N_{\text {eff }} / \bar{k} L$ in Eq. (46) now becomes $O(\delta k L)^{2}$ instead of order unity. The growth of $N_{\text {eff }}$ with increasing $\delta k L$ is shown in Fig. 6 in the special case of a disk geometry, for two values of $\bar{k} L$. Similar results are obtained for other geometries.

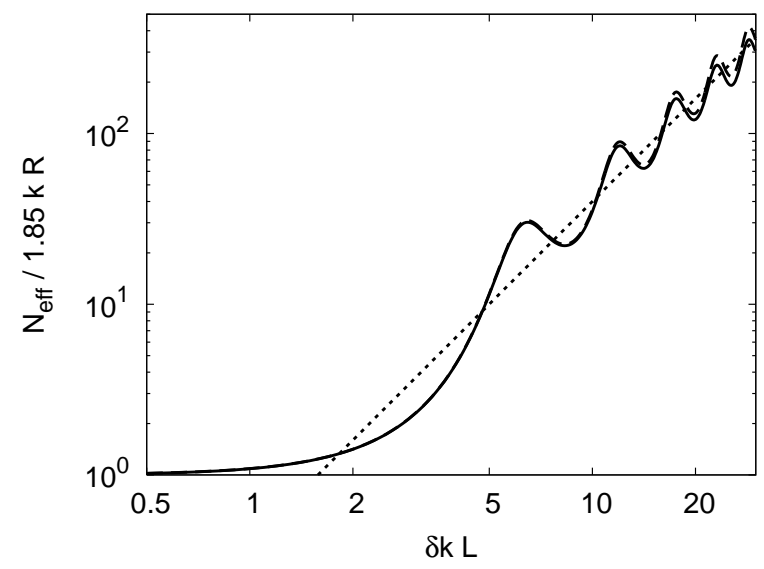

FIG. 6: The factor $N_{\text {eff }}$, which governs the reduction of the covariance $\overline{\delta v_{\alpha \beta} \delta v_{\alpha \gamma}}$ as compared with the variance $\overline{\delta v_{\alpha \beta}^{2}}$, is computed for a disk geometry in accordance with the definitions (44) and (45). $N_{\text {eff }}$ is computed as a function of $\bar{k} L$ and $\delta k L$, where $\bar{k}=\left(k_{\beta}+k_{\gamma}\right) / 2$, and $\delta k=\left|k_{\beta}-k_{\gamma}\right|$. The solid curve corresponds to $\bar{k} L=30$ and the dashed curve to $\bar{k} L=70$. The dotted line shows the quadratic scaling with $\delta k L$, as predicted by Eq. (47).

Eq. (46) then becomes

$$
\overline{\delta v_{\alpha \beta} \delta v_{\alpha \gamma}} \sim-\Delta^{2}\left(\frac{2}{\beta}\right)^{2} \frac{\ln \bar{k} L+b_{g}}{(\bar{k} L)^{3}(\delta k L)^{2}} .
$$

In Fig. 7 we show the dependence of the diagonal interaction matrix element covariance on $\delta k L$ for a disk at fixed $\bar{k} L=60$. The numerical covariance of the random wave model (solid line) is in reasonable agreement with the RMT expression (46) (dashed line).

\section{ONE-BODY MATRIX ELEMENTS}

When an electron is added to the finite dot, charge accumulates on the surface and its effect can be described by a one-body potential energy $\mathcal{V}(\vec{r})$. For an elliptical $2 \mathrm{D}$ geometry, the variation in this mean field potential energy due to the addition of an electron is given by

$$
\mathcal{V}(x, y)=-\frac{\Delta / 4}{\sqrt{1-x^{2} / a^{2}-y^{2} / b^{2}}},
$$

where we have used the Thomas-Fermi screening length to obtain the scale of $\mathcal{V}$.

We note that for non-elliptical shapes, the correct form of $\mathcal{V}(\vec{r})$ may be calculated numerically. However, we will see below that the fluctuations of its matrix elements

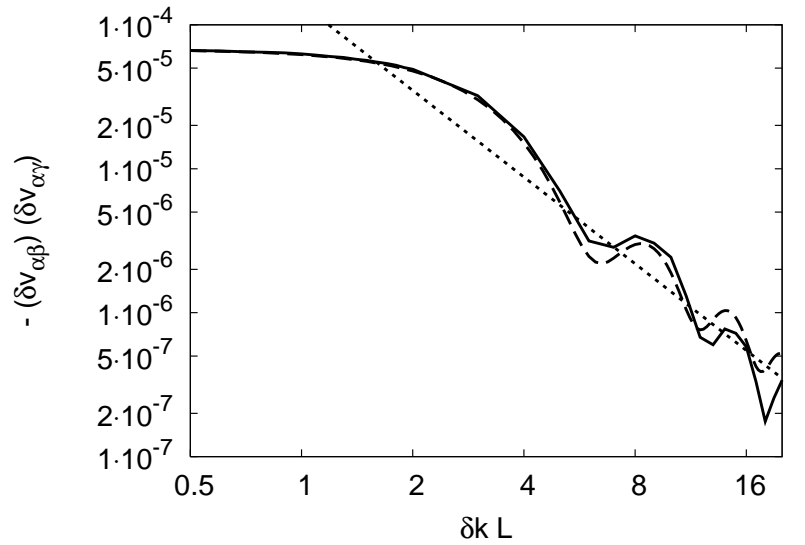

FIG. 7: The diagonal interaction matrix element covariance $\overline{\delta v_{\alpha \beta} \delta v_{\alpha \gamma}}$ for $\delta k \equiv k_{\beta}-k_{\gamma} \neq 0$ is plotted versus $\delta k L \sim \delta E / E_{T}$ for real random waves inside a disk; $\bar{k} L=\left(k_{\beta}+k_{\gamma}\right) L / 2$ is fixed at 60 . The numerical result obtained by substituting Eq. (36) (with $A_{\mu \mu^{\prime}}$ now given by Eq. (45)) into Eq. (33) (solid line) is compared with the the analytic RMT approximation of Eq. (46) (dashed line). The dotted line describes the scaling $\overline{\delta v_{\alpha \beta} \delta v_{\alpha \gamma}} \sim(\delta k L)^{-2}$ of Eq. (48), valid for $\delta k L \gg 1$.

depend only weakly on the precise form of the potential, and are instead dominated by the overall normalization and the $1 / \sqrt{d}$ divergence near the boundary.

The diagonal matrix elements of $\mathcal{V}(\vec{r})$ are given by

$$
v_{\alpha} \equiv \mathcal{V}_{\alpha \alpha}=\int_{V} d \vec{r}\left|\psi_{\alpha}(\vec{r})\right|^{2} \mathcal{V}(\vec{r})
$$

Using $\overline{\left|\psi_{\alpha}(\vec{r})\right|^{2}}=1 / V$, we find $\overline{v_{\alpha}}=\frac{1}{V} \int_{V} d \vec{r} \mathcal{V}(\vec{r}) \equiv \overline{\mathcal{V}}=$ $-\Delta / 2$.

Again, of main interest are the fluctuations in $v_{\alpha}$. As in Section IVA, we begin by expressing the variance in terms of the wave function intensity correlator [20, 21]

$$
\begin{aligned}
\overline{\delta v_{\alpha}^{2}} & =\int_{V} \int_{V} d \vec{r} d \vec{r}^{\prime} \mathcal{V}(\vec{r}) \tilde{C}\left(\vec{r}, \vec{r}^{\prime}\right) \mathcal{V}\left(\vec{r}^{\prime}\right) \\
& =\int_{V} \int_{V} d \vec{r} d \vec{r}^{\prime} \tilde{\mathcal{V}}(\vec{r}) C\left(\vec{r}, \vec{r}^{\prime}\right) \tilde{\mathcal{V}}\left(\vec{r}^{\prime}\right),
\end{aligned}
$$

where $\tilde{\mathcal{V}}=\mathcal{V}-\overline{\mathcal{V}}$. Because only one power of $C$ or $\tilde{C}$ appears in the above expressions, the integral is dominated by distant pairs of points $\left|\vec{r}-\vec{r}^{\prime}\right| \sim L$, and scales as $1 / k L$ :

$$
\overline{\delta v_{\alpha}^{2}}=\frac{c_{g}}{\beta} \frac{\Delta^{2}}{k L}+O\left(\frac{\Delta^{2}}{(k L)^{2}}\right),
$$

where $c_{g}$ is a shape-dependent dimensionless coefficient.

In Fig. 8 we show the result of integrating Eq. (51) for a disk with $\beta=1$. The solid lower curve corresponds to the analytic potential of Eq. (49) while the dashed lower curve corresponds to the "schematic" potential

$$
\mathcal{V}_{\mathrm{sch}}(\vec{r}) \sim\left(\min _{\vec{R} \in \mathcal{C}}|\vec{r}-\vec{R}|\right)^{-\frac{1}{2}},
$$




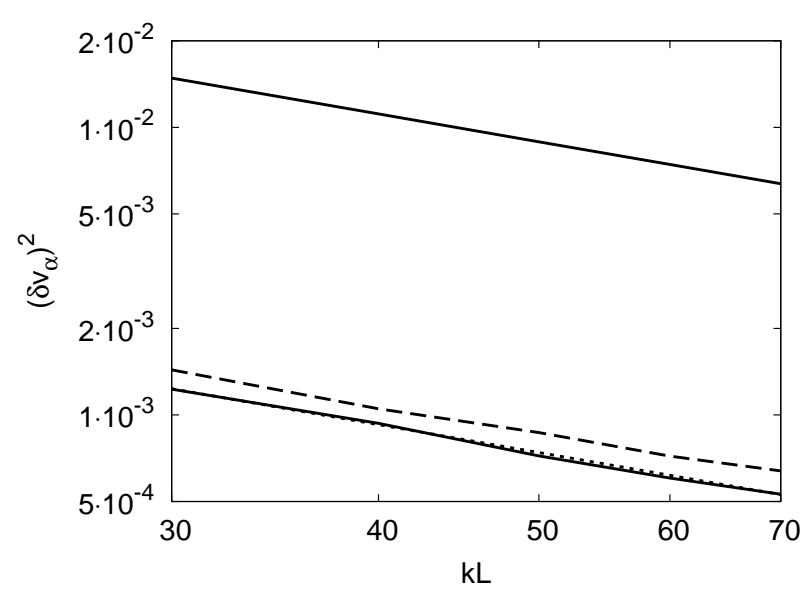

FIG. 8: The expression (51) for the one-body matrix element variance $\overline{\delta v_{\alpha}^{2}}$ is plotted as a function of $k L$ for real random waves inside a disk using the analytic potential of Eq. (49) (lower solid curve) and the schematic potential of Eq. (54) (lower dashed curve). The dotted line, which is almost indistinguishable from the lower solid curve, is the power-law prediction of Eq. (53). The upper solid curve is calculated from an unsubtracted integral, where the unnormalized propagator $C\left(\vec{r}, \vec{r}^{\prime}\right)$ is substituted for $\tilde{C}\left(\vec{r}, \vec{r}^{\prime}\right)$ in Eq. (51).

which has the same singularity at the boundary $\mathcal{C}$ as the true potential (49) and is scaled to have the same average as $\mathcal{V}(\vec{r})$. The upper solid curve is obtained by substituting the unnormalized correlator $C\left(\vec{r}, \vec{r}^{\prime}\right)$ in place of $\tilde{C}\left(\vec{r}, \vec{r}^{\prime}\right)$ in Eq. (51). We note that normalization, which had only a moderate effect on the two-body matrix element fluctuations, here reduces the variance by a full order of magnitude, resulting in a very small prefactor $c_{g}$ in Eq. (53). (For a disk, $c_{g}=0.035$, and the power-law prediction of Eq. (53) is indicated in Fig. 8 8 as a dotted line.) This reduction in the variance is due to the fact that after normalization, according to Eq. (19), the one-body integrand (51) ceases to be positive everywhere, resulting in substantial cancellation in the integral. This is in contrast with the two-body integrand in Eq. (27), which remains positive even after normalization. On the other hand, the difference between the real and the schematic potential after normalization is only an $18 \%$ effect. This fact is useful for studying one-body matrix elements in chaotic geometries, where no analytic form exists for the surface-charge potential.

The small value of the coefficient $c_{g}$ leads to values of $\overline{\delta v_{\alpha}^{2}}$ (see Fig. 8) that are numerically smaller in the physically interesting $k L$ regime than the corresponding values of the two-body matrix element variance $\overline{\delta v_{\alpha \beta}^{2}}$ (see Fig. 2), despite the fact that the former is parametrically larger in a $1 / k L$ expansion.

The two lower curves in Fig. 3 show the dependence of $\overline{\delta v_{\alpha}^{2}}$ on the dot's geometry. We find the one-body matrix element variance to have much stronger shape dependence than the two-body matrix element variance.
This is due mostly to the fact that here the coefficient of the leading term in Eq. (53) is already shape dependent, in contrast with the shape-independence of the leading logarithmic term in the two-body expression (26). In particular, an aspect ratio of $r=4$ results in a $14 \%$ reduction in the coefficient $c_{g}$ in Eq. (53). We also note the divergence between the $k L=30$ and $k L=70$ curves for $r>4$, indicating that for very large aspect ratios, the simple inverse relationship between $k L$ and the variance breaks down in the energy range of interest and the subleading terms in Eq. (53) acquire greater importance.

\section{MATRIX ELEMENT DISTRIBUTIONS}

The central limit theorem implies that all interaction matrix elements such as $v_{\alpha \beta}, v_{\alpha \alpha}, v_{\alpha \beta \gamma \delta}$, and $v_{\alpha}$ in the random wave model must be distributed as Gaussian random variables as $k L \rightarrow \infty$, since each of them is defined as an integral over a large volume of a random integrand with decaying correlations. This justifies our focus so far on the variance and covariance of these matrix elements. However, we have seen above that non-universal finite $k L$ effects can be significant in the experimentally relevant regime $k L \leq 70$, most notably for the variance ratios shown in Fig. 4. Here we look for finite- $k L$ effects on the shape of matrix element distributions.

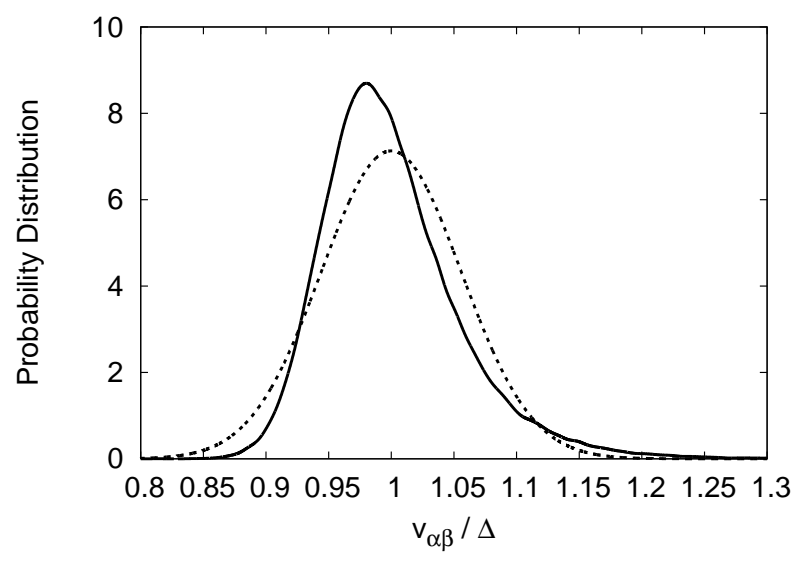

FIG. 9: The distribution of diagonal interaction matrix elements $v_{\alpha \beta}$ is shown for normalized real random waves in a disk at $k L=70$ (solid curve). A Gaussian distribution with the same mean and variance is shown as a dotted curve for comparison.

The distribution of diagonal interaction matrix element $v_{\alpha \beta}$ is shown in Fig. 9 for normalized random waves in a disk (solid curve). Results for other dot's geometries and for other matrix elements (i.e., $v_{\alpha \alpha}, v_{\alpha \beta \gamma \delta}$, and $v_{\alpha}$ ) are qualitatively similar. We observe that the distribution has a long tail on the right side as compared with a Gaussian distribution of the same mean and variance (dotted curve). In other words, there is an excess of 
anomalously large matrix elements, compensated for by a reduction in the median to a value slightly below $\Delta$. Indeed, the right tail has a shape closer to an exponential than to a Gaussian, reminiscent of the distribution of many-body matrix elements in realistic atomic shell model calculations 34].

Deviations from a Gaussian shape can be quantified by considering higher moments such as the skewness

$$
\gamma_{1}=\frac{\overline{\delta v_{\alpha \beta}^{3}}}{\left[\overline{\delta v_{\alpha \beta}^{2}}\right]^{3 / 2}}
$$

and excess kurtosis

$$
\gamma_{2}=\frac{\overline{\delta v_{\alpha \beta}^{4}}-3\left[\overline{\delta v_{\alpha \beta}^{2}}\right]^{2}}{\left[\overline{\delta v_{\alpha \beta}^{2}}\right]^{2}}
$$

For large $k L$, we may estimate these higher moments in a manner analogous to our estimate for the variance in Eq. (24). Specifically,

$$
\begin{gathered}
\overline{\delta v_{\alpha \beta}^{3}} \equiv \\
\equiv \Delta^{3} V^{3} \overline{\left[\int_{V} d \vec{r}\left|\psi_{\alpha}(\vec{r})\right|^{2}\left|\psi_{\beta}(\vec{r})\right|^{2}-\overline{\int_{V} d \vec{r}\left|\psi_{\alpha}(\vec{r})\right|^{2}\left|\psi_{\beta}(\vec{r})\right|^{2}}\right]^{3}} \\
=\Delta^{3} V^{3}\left\{\int_{V} \int_{V} \int_{V} d \vec{r} d \vec{r}^{\prime} d \vec{r}^{\prime \prime}\right. \\
\frac{\left[\left(\left|\psi_{\alpha}(\vec{r})\right|^{2}-\frac{1}{V}\right)\left(\left|\psi_{\alpha}\left(\vec{r}^{\prime}\right)\right|^{2}-\frac{1}{V}\right)\left(\left|\psi_{\alpha}\left(\vec{r}^{\prime \prime}\right)\right|^{2}-\frac{1}{V}\right)\right]}{\left[\left(\left|\psi_{\beta}(\vec{r})\right|^{2}-\frac{1}{V}\right)\left(\left|\psi_{\beta}\left(\vec{r}^{\prime}\right)\right|^{2}-\frac{1}{V}\right)\left(\left|\psi_{\beta}\left(\vec{r}^{\prime \prime}\right)\right|^{2}-\frac{1}{V}\right)\right]} \\
+\Delta^{3} V^{3} \int_{V} \int_{V} \int_{V} d \vec{r} d \vec{r}^{\prime} d \vec{r}^{\prime \prime} \frac{u_{\alpha}(\vec{r}) u_{\alpha}\left(\vec{r}^{\prime}\right) u_{\alpha}\left(\vec{r}^{\prime \prime}\right)}{2}
\end{gathered}
$$

where $u_{\alpha}(\vec{r})=\left|\psi_{\alpha}(\vec{r})\right|^{2}-\frac{1}{V}$ is the excess intensity. The ellipsis in Eq. (57) indicates omitted terms involving the intensity correlation of two different wave functions at different points, such as the correlator $C_{2}\left(\vec{r}, \vec{r}^{\prime}\right)$ defined in Eq. (25) and its generalization to three distinct points.

The three-point intensity correlator for a single random wave function can be computed to leading order in $k L$ by following the same procedure that led to the leadingorder two-point intensity correlator (12): performing all possible contractions to rewrite the quantity of interest as a product of amplitude correlators and making use of Eq. (11). Thus

$$
\begin{aligned}
& \overline{u_{\alpha}(\vec{r}) u_{\alpha}\left(\vec{r}^{\prime}\right) u_{\alpha}\left(\vec{r}^{\prime \prime}\right)}= \\
= & \overline{\left(|\psi(\vec{r})|^{2}-\frac{1}{V}\right)\left(\left|\psi\left(\vec{r}^{\prime}\right)\right|^{2}-\frac{1}{V}\right)\left(\left|\psi\left(\vec{r}^{\prime \prime}\right)\right|^{2}-\frac{1}{V}\right)} \\
\approx & c_{3 \beta} \overline{\psi^{*}(\vec{r}) \psi\left(\vec{r}^{\prime}\right)} \frac{\psi^{*}\left(\vec{r}^{\prime}\right) \psi\left(\vec{r}^{\prime \prime}\right)}{\psi^{*}\left(\vec{r}^{\prime \prime}\right) \psi(\vec{r})} \\
= & \frac{c_{3 \beta}}{V^{3}} J_{0}\left(k\left|\vec{r}-\vec{r}^{\prime}\right|\right) J_{0}\left(k\left|\vec{r}^{\prime}-\vec{r}^{\prime \prime}\right|\right) J_{0}\left(k\left|\vec{r}^{\prime \prime}-\vec{r}\right|\right)
\end{aligned}
$$

where $c_{31}=8$ for real waves and $c_{32}=2$ for complex waves are combinatorial factors. Normalization effects analogous to those included in Eq. (19) are subleading in this case, since $\overrightarrow{u_{\alpha}(\vec{r}) u_{\alpha}\left(\vec{r}^{\prime}\right) u_{\alpha}\left(\vec{r}^{\prime \prime}\right)}$ is a fluctuating quantity with average close to zero instead of being everywhere positive. The result (58) scales as $V^{-3}(k L)^{-3 / 2}$ in the typical case when the inter-point separations are all of order $L$.

Combining Eq. (58) with Eq. (57), we have

$$
\begin{aligned}
\overline{\delta v_{\alpha \beta}^{3}}= & c_{3 \beta}^{2}\left(\frac{\Delta}{V}\right)^{3} \int_{V} \int_{V} \int_{V} d \vec{r} d \vec{r}^{\prime} d \vec{r}^{\prime \prime}\left\{J_{0}^{2}\left(k\left|\vec{r}-\vec{r}^{\prime}\right|\right)\right. \\
& \left.\times J_{0}^{2}\left(k\left|\vec{r}^{\prime}-\vec{r}^{\prime \prime}\right|\right) J_{0}^{2}\left(k\left|\vec{r}^{\prime \prime}-\vec{r}\right|\right)\right\} \\
= & b_{3 g} c_{3 \beta}^{2} \frac{\Delta^{3}}{(k L)^{3}}
\end{aligned}
$$

where $b_{3 g}$ is a shape-dependent constant analogous to $b_{g}$ in the calculation of the variance (28). In contrast to the variance calculation (26), here we have no large shortdistance contribution resulting in a logarithmic contribution at leading order. The irrelevance of the shortdistance contribution may be seen by noting that a fraction $\sim \epsilon^{4}$ of the integration space in Eq. (59) has points $\vec{r}, \vec{r}^{\prime}$, and $\vec{r}^{\prime \prime}$ all within distance $\epsilon$ of one another, while the integrand is only enhanced by a factor $\sim \epsilon^{-3}$ in this region. For a disk geometry, $b_{3 g}=1.3$.

Similarly, the fourth moment $\overline{\delta v_{\alpha \beta}^{4}}$ involves the fourpoint intensity correlation function, which to leading order in $1 / k L$ takes the form

$$
\begin{aligned}
& \overline{u_{\alpha}(\vec{r}) u_{\alpha}\left(\vec{r}^{\prime}\right) u_{\alpha}\left(\vec{r}^{\prime \prime}\right) u_{\alpha}\left(\vec{r}^{\prime \prime \prime}\right)} \approx \\
& c_{4 \beta}\left[J_{0}\left(k\left|\vec{r}-\vec{r}^{\prime}\right|\right) J_{0}\left(k\left|\vec{r}^{\prime}-\vec{r}^{\prime \prime}\right|\right) J_{0}\left(k\left|\vec{r}^{\prime \prime}-\vec{r}^{\prime \prime \prime}\right|\right) J_{0}\left(k\left|\vec{r}^{\prime \prime \prime}-\vec{r}\right|\right)\right. \\
& \left.+\left(\vec{r}^{\prime} \leftrightarrow \vec{r}^{\prime \prime}\right)+\left(\vec{r}^{\prime \prime} \leftrightarrow \vec{r}^{\prime \prime \prime}\right)\right] \\
& +\left(\frac{2}{\beta}\right)^{2}\left[J_{0}^{2}\left(k\left|\vec{r}-\vec{r}^{\prime \prime}\right|\right) J_{0}^{2}\left(k\left|\vec{r}^{\prime}-\vec{r}^{\prime \prime \prime}\right|\right)\right. \\
& \left.+\left(\vec{r}^{\prime} \leftrightarrow \vec{r}^{\prime \prime}\right)+\left(\vec{r}^{\prime \prime} \leftrightarrow \vec{r}^{\prime \prime \prime}\right)\right] .
\end{aligned}
$$

Here $c_{41}=16$ and $c_{42}=2$ are combinatorial factors. Performing a four-fold integral over volume, we obtain

$$
\begin{aligned}
\overline{\delta v_{\alpha \beta}^{4} \approx} & \Delta^{4} V^{4} \int_{V} \int_{V} \int_{V} \int_{V} d \vec{r} d \vec{r}^{\prime} d \vec{r}^{\prime \prime} d \vec{r}^{\prime \prime \prime} \\
& \times{\overline{u_{\alpha}(\vec{r}) u_{\alpha}\left(\vec{r}^{\prime}\right) u_{\alpha}\left(\vec{r}^{\prime \prime}\right) u_{\alpha}\left(\vec{r}^{\prime \prime \prime}\right)}}^{2} \\
\approx & 3\left(c_{4 \beta}^{2}+\left(\frac{2}{\beta}\right)^{4}\right)\left(\frac{\Delta}{V}\right)^{4} \int_{V} \int_{V} \int_{V} \int_{V} d \vec{r} d \vec{r}^{\prime} d \vec{r}^{\prime \prime} d \vec{r}^{\prime \prime \prime} \\
& {\left[J_{0}^{2}\left(k\left|\vec{r}-\vec{r}^{\prime}\right|\right) J_{0}^{2}\left(k\left|\vec{r}^{\prime}-\vec{r}^{\prime \prime}\right|\right)\right.} \\
& \left.\times J_{0}^{2}\left(k\left|\vec{r}^{\prime \prime}-\vec{r}^{\prime \prime \prime}\right|\right) J_{0}^{2}\left(k\left|\vec{r}^{\prime \prime \prime}-\vec{r}\right|\right)\right]+3{\overline{\delta v_{\alpha \beta}^{2}}}^{2},
\end{aligned}
$$

where in the second step we have omitted terms in the integrand containing odd powers of $J_{0}$ (these terms have oscillating sign and contribute to the integral only at subleading order in $k L$ ). We have also separated out a term proportional to the square of the variance (24); this term 
corresponds to disconnected diagrams and does not contribute to the fourth cumulant or the excess kurtosis $\gamma_{2}$. The final result is

$$
\overline{\delta v_{\alpha \beta}^{4}}-3{\overline{\delta v_{\alpha \beta}^{2}}}^{2}=3 b_{4 g}\left(c_{4 \beta}^{2}+\left(\frac{2}{\beta}\right)^{4}\right) \frac{\Delta^{4}}{(k L)^{4}},
$$

where the shape-dependent coefficient $b_{4 g}=1.0$ for a disk geometry. Higher-order cumulants beyond (60) and (63) may be computed similarly. It is evident from the preceding discussion that the $n$-th cumulant involves an $n$-fold integral of a product of $2 n$ Bessel functions and scales as $\Delta^{n} /(k L)^{n}$, with a combinatorial $\beta$-dependent prefactor and a geometry-dependent overall dimensionless constant.

When the cumulants $\overline{\delta v_{\alpha \beta}^{3}}$ and $\overline{\delta v_{\alpha \beta}^{4}}-3{\overline{\delta v_{\alpha \beta}^{2}}}^{2}$ for normalized random waves in a disk are computed numerically, they compare well with the power-law predictions of (60) and (63) at sufficiently large $k L$ (not shown).

Combining Eqs. (60) and (63) with our previous result (26) for the variance, we find the skewness

$$
\gamma_{1}=b_{3 g} c_{3 \beta}^{2}\left(\frac{\beta}{2}\right)^{3}\left(\frac{\pi}{3}\right)^{3 / 2}(\ln k L)^{-3 / 2}
$$

and excess kurtosis

$$
\gamma_{2}=b_{4 g}\left(c_{4 \beta}^{2}+\left(\frac{2}{\beta}\right)^{4}\right)\left(\frac{\pi^{2}}{3}\right)(\ln k L)^{-2}
$$

at large $k L$. Because the decay is only logarithmic, $\gamma_{1}$ and $\gamma_{2}$ never become small for values of $k L$ relevant in the experiments, as seen in Fig. 10. The same holds for the higher moments. Therefore, the random wave model leads to strongly non-Gaussian matrix element distributions.

Higher cumulants for the distribution of doublediagonal interaction matrix elements $v_{\alpha \alpha}$ and for offdiagonal matrix elements $v_{\alpha \beta \gamma \delta}$ may be obtained similarly; only the $\beta$-dependent combinatorial prefactors in Eqs. (60) and (63) are modified and the $n$-th cumulants again scale as $\Delta^{n} /(k L)^{n}$. Of course, the skewness $\gamma_{1}$ and all odd moments vanish identically for $v_{\alpha \beta \gamma \delta}$, since the distribution in this case is manifestly symmetric around zero.

Finally, we find that the distribution of one-body matrix elements $v_{\alpha}$ approaches more rapidly a Gaussian form, with skewness and excess kurtosis decaying to zero as a power law instead of a logarithm in $k L$. For example, to leading order in $1 / k L$,

$$
\begin{aligned}
\overline{\delta v_{\alpha}^{3}} & =\frac{1}{V^{3}} \int_{V} \int_{V} d \vec{r} d \vec{r}^{\prime} d \vec{r}^{\prime \prime}\left[\mathcal{V}(\vec{r}) J_{0}\left(k\left|\vec{r}-\vec{r}^{\prime}\right|\right) \mathcal{V}\left(\vec{r}^{\prime}\right)\right. \\
& \left.\times J_{0}\left(k\left|\vec{r}-\vec{r}^{\prime \prime}\right|\right) \mathcal{V}\left(\vec{r}^{\prime \prime}\right) J_{0}\left(k\left|\vec{r}^{\prime \prime}-\vec{r}\right|\right)\right] \sim \frac{\Delta^{3}}{(k L)^{2}}(66)
\end{aligned}
$$

and comparing with Eq. (53) we find

$$
\gamma_{1}^{\prime} \sim(k L)^{-1 / 2}
$$

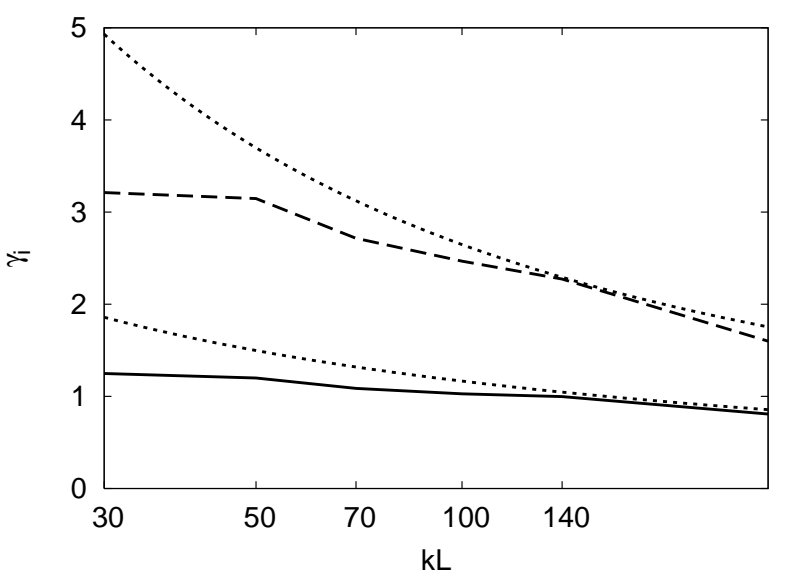

FIG. 10: The skewness $\gamma_{1}$ (55), indicated by a solid curve, and excess kurtosis $\gamma_{2}$ (56), indicated by a dashed curve, for the distribution of diagonal interaction matrix elements $v_{\alpha \beta}$ in a disk are computed within the normalized real random wave model. Analytic predictions (64) and (65) valid at large $k L$ are indicated by dotted lines.

where $\gamma_{1}^{\prime}$ is defined as in (55) with $v_{\alpha}$ replacing $v_{\alpha \beta}$. At the experimentally relevant values of $k L$, the deviation from Gaussian behavior for one-body matrix elements is nevertheless significant, though it is less pronounced than for two-body matrix elements. Even at $k L=70$, we have $\gamma_{1}^{\prime} \approx \gamma_{2}^{\prime} \approx 0.6$

\section{SUMMARY AND CONCLUSION}

We have studied fluctuations of two-body interaction matrix elements and surface charge one-body matrix elements in ballistic quantum dots as a function of semiclassical parameter $k L$. Understanding the quantitative behavior of these fluctuations is important for a proper analysis of peak spacing statistics and scrambling effects in the Coulomb blockade regime.

The variance and higher cumulants of two-body and one-body matrix elements can be derived from spatial correlations of the single-particle Hartree-Fock wave functions. For a chaotic dot, we have estimated these correlations to leading order in $k L$ using Berry's random wave model. The variances of two-body matrix elements are found to scale as $\ln k L /(k L)^{2}$, with universal prefactors that depend only on the symmetry class of the system. Geometry-dependent effects on the variance enter at the order of $1 /(k L)^{2}$, where the random wave intensity correlator must be corrected to satisfy individual wave function normalization in a finite volume. To understand such corrections we have studied a normalized random wave model. Variance ratios such as $\overline{\delta v_{\alpha \alpha}^{2}} / \overline{\delta v_{\alpha \beta}^{2}}$ converge only with a logarithmic rate in the $k L \rightarrow \infty$ limit. As a result, the asymptotic values of such ratios are not yet reached in the regime of experimental inter- 
est. The interaction matrix element covariance is important in calculations of spectral scrambling; this quantity, which must be negative on average due to a sum rule, is computed as a function of energy separation using the intensity correlator between two orthonormal random wave functions.

The variance of one-body matrix elements $v_{\alpha}$ (of, e.g., the surface charge potential) is affected by normalization even at leading order, resulting in $O(1 / k L)$ scaling in a random wave model, with a shape-dependent prefactor that may be computed using the normalized intensity correlator. For typical experimental values of $k L$, we find the distributions of two-body and one-body matrix elements to be strongly non-Gaussian. Thus, higher cumulants of these matrix elements may play a role in the peak spacing statistics, especially in the case of two-body matrix elements where we have seen that the approach to a Gaussian distribution is logarithmically slow.

The absence of bimodality in the measured peak spacing distribution at low temperatures cannot be explained by the exchange interaction alone and must originate in the non-universal part of the electron-electron interac- tion. Sufficiently large fluctuations of such interaction matrix elements are required to wash out the bimodality of the peak spacing distribution. The fluctuation width estimates derived here in the framework of the random wave model are too small. It is therefore necessary to study interaction matrix element fluctuations in real chaotic systems and in system with mixed dynamics, where dynamical effects may lead to enhanced fluctuations 35]. Such studies are important for the quantitative understanding of spectral scrambling and the measured peak spacing distribution.

\section{Acknowledgments}

We acknowledge useful discussions with Y. Gefen, Ph. Jacquod, and C. H. Lewenkopf. This work was supported in part by the U.S. Department of Energy Grants No. DE-FG03-00ER41132 and DE-FG-0291-ER40608 and by the National Science Foundation under Grant No. PHY-0545390.
[1] Y. Alhassid, Rev. Mod. Phys. 72, 895 (2000).

[2] T. Guhr, A. Müller-Groeling, and H. A. Weidenmüller, Phys. Rep. 299, 189 (1998).

[3] R. A. Jalabert, A. D. Stone, and Y. Alhassid, Phys. Rev. Lett. 68, 3468 (1992).

[4] Y. Alhassid and H. Attias, Phys. Rev. Lett. 76, 1711 (1996).

[5] Y. Alhassid, Phys. Rev. B 58, R 13383 (1998).

[6] J. A. Folk, S. R. Patel, S. F. Godijn, A. G. Huibers, S. M. Cronenwett, C. M. Marcus, K. Campman, and A. C. Gossard, Phys. Rev. Lett. 76, 1699 (1996).

[7] A. M. Chang, H. U. Baranger, L. N. Pfeiffer, K. W. West, and T. Y. Chang, Phys. Rev. Lett. 76, 1695 (1996).

[8] J. A. Folk, C. M. Marcus, and J. S. Harris, Jr., Phys. Rev. Lett. 87, 206802 (2001).

[9] U. Sivan, R. Berkovits, Y. Aloni, O. Prus, A. Auerbach, and G. Ben-Yoseph, Phys. Rev. Lett. 77, 1123 (1996).

[10] F. Simmel, T. Heinzel, and D. A. Wharam, Europhys. Lett. 38, 123 (1997).

[11] S. R. Patel, S. M. Cronenwett, D. R. Stewart, A. G. Huibers, C. M. Marcus, C. I. Duruöz, J. S. Harris, Jr., K. Campman, and A. C. Gossard, Phys. Rev. Lett. 80, 4522 (1998).

[12] S. Lüscher, T. Heinzel, K. Ensslin, W. Wegscheider, and M. Bichler, Phys. Rev. Lett. 86, 2118 (2001).

[13] I. L. Kurland, I. L. Aleiner, and B. L. Altshuler, Phys. Rev. B 62, 14886 (2000).

[14] I. L. Aleiner, P. W. Brouwer, and L. I. Glazman, Phys. Rep. 358, 309 (2002).

[15] Y. Alhassid and T. Rupp, Phys. Rev. Lett. 91, 056801 (2003).

[16] G. Usaj and H. U. Baranger, Phys. Rev. B 67, 121308(R) (2003).

[17] Ya. M. Blanter, A. D. Mirlin, and B. A. Muzykantskii,
Phys. Rev. Lett. 78, 2449 (1997).

[18] Y. Alhassid, H. A. Weidenmüller, and A. Wobst, Phys. Rev. B 72, 045318 (2005).

[19] T. Koopmans, Physica (Amsterdam) 1, 104 (1934).

[20] Y. Alhassid and S. Malhotra, Phys. Rev. B 66, 245313 (2002).

[21] G. Usaj and H. U. Baranger, Phys. Rev. B 66, 155333 (2002).

[22] A. D. Mirlin, Phys. Rep. 326, 259 (2000).

[23] M. V. Berry, J. Phys. A 10, 2083 (1977).

[24] Y. Alhassid and Y. Gefen, cond-mat/0101461.

[25] Y. Alhassid, H. A. Weidenmüller, and A. Wobst, Phys. Rev. B 76, 193110 (2007).

[26] I. V. Gornyi and A. D. Mirlin, Phys. Rev. E 65, 025202(R) (2002); J. Low Temp. Phys. 126, 1339 (2002).

[27] G. Murthy, R. Shankar, and H. Mathur, Phys. Rev. B 72, 075364 (2005).

[28] J. D. Urbina and K. Richter, Eur. Phys. J. Special Topics 145, 255 (2007).

[29] S. Goldstein, J. L. Lebowitz, R. Tumulka, and N. Zanghì, J. Stat. Phys. 125, 1193 (2006).

[30] B. L. Altshuler, Y. Gefen, A. Kamenev, and L. S. Levitov, Phys. Rev. Lett. 78, 2803 (1997).

[31] D. Ullmo and H. U. Baranger, Phys. Rev. B 64, 245324 (2001).

[32] Since the shape dependence is observed to be very weak within the random wave model, the values of $b_{g}^{\prime}$ and $b_{g}^{\prime \prime}$ for other geometries are very similar.

[33] L. A. Bunimovich, Commun. Math. Phys. 65, 295 (1979).

[34] V. V. Flambaum, A. A. Gribakina, G. F. Gribakin, and M G. Kozlov, Phys. Rev. A 50, 267 (1994).

[35] D. Ullmo, T. Nagano, and S. Tomsovic, Phys. Rev. Lett 90, 176801 (2003); 91, 179901(E) (2003). 